\title{
Complex Thoraco-Abdominal Trauma: Transdiaphragmatic Inter- costal Hernia and Rib Fractures
}

\author{
Sally Shepherd, $M D^{1^{*}}$, Annelise Cocco, MBBS, FRACS ${ }^{1}$, Phillip Antippa, MBBS, FRACS ${ }^{2}$ \\ and Jacob McCormick, MBBS, FRACS ${ }^{1}$
}

${ }^{1}$ Department of General Surgery, The Royal Melbourne Hospital, Australia

${ }^{2}$ Department of Cardiothoracic Surgery, The Royal Melbourne Hospital, Australia

*Corresponding author: Dr. Sally Shepherd, Department of General Surgery, The Royal Melbourne Hospital, Level 6 East, 300 Grattan Street Parkville VIC 3050, Australia, Tel: 0415089244

\begin{abstract}
Transdiaphragmatic intercostal herniation of abdominal contents into the chest is rare. In this case, a combined thoracic and abdominal approach allowed reduction of the small bowel hernia, repair of the diaphragmatic defect and reconstruction of the chest wall.
\end{abstract}

\section{Keywords}

Intercostal hernia, Rib fracture, Trauma, Transdiaphragmatic

\section{Introduction}

Injuries to the costal margin which involve herniation of abdominal contents into the chest are rare. Two major mechanisms of transdiaphragmatic intercostal hernia are recognised: post-traumatic and spontaneous. It is hypothesised that disruption of either the diaphragm or intercostal muscles leads to herniation of the abdominal contents, and that negative intra-thoracic pressure then draws the herniated viscera further into the chest [1].

Unlike the specific entity of transdiaphragmatic intercostal hernia, there are many case reports and case series outlining the identification and management of a diaphragmatic rupture. Diaphragmatic injury is known to occur in 1.4 to $8 \%$ of patients following blunt trauma, and commonly occurs in association with solid organ injury (as was also seen in our patient) [2]. Rib fractures are present in association with a diaphragmatic injury in approximately $50 \%$ of cases [3]. The association of rib fractures with trans- diaphragmatic intercostal hernia has not been quantified.

\section{Case Description}

A 66-year-old male was transferred to the emergency department at an Australian Level 1 Trauma Centre following a high speed motor vehicle crash. He had a significant past medical history of coronary artery disease requiring a coronary artery bypass graft, type 2 diabetes and asthma. Upon initial ATLS assessment, respiratory distress was treated with high flow supplemental oxygen.

The patient's chest X-Ray (CXR) demonstrated fractures of the right $3^{\text {rd }}-8^{\text {th }}$ ribs and left $4^{\text {th }}-8^{\text {th }}$ ribs inclusive. Computerised tomography (CT) revealed bilateral rib fractures with associated flail segment and significantly displaced fractures of the right $3^{\text {rd }}$ to $5^{\text {th }}$ ribs; a right diaphragmatic laceration; grade 2 liver injury; and transdiaphragmatic small bowel herniation (Figure 1).

Subsequently the patient developed type 2 respiratory failure and was intubated. On day two post-admission, following respiratory optimisation in the intensive care unit (ICU), the patient was transferred to the operating theatre. He underwent a laparoscopic reduction of the diaphragmatic hernia and primary suture repair of the defect using a 2-0 non-absorbable suture and placement of a composite mesh secured with tacking devices. On completion of the abdominal component of the procedure, the thoracic team performed surgical stabilisation of the right

Citation: Shepherd S, Cocco A, Antippa P, McCormickJ (2019) Complex Thoraco-Abdominal Trauma: Transdiaphragmatic Intercostal Hernia and Rib Fractures. Trauma Cases Rev 5:079. doi.org/10.23937/24695777/1510079

Accepted: October 12, 2019: Published: October 14, 2019

Copyright: (C) 2019 Shepherd S, et al. This is an open-access article distributed under the terms of the Creative Commons Attribution License, which permits unrestricted use, distribution, and reproduction in any medium, provided the original author and source are credited. 

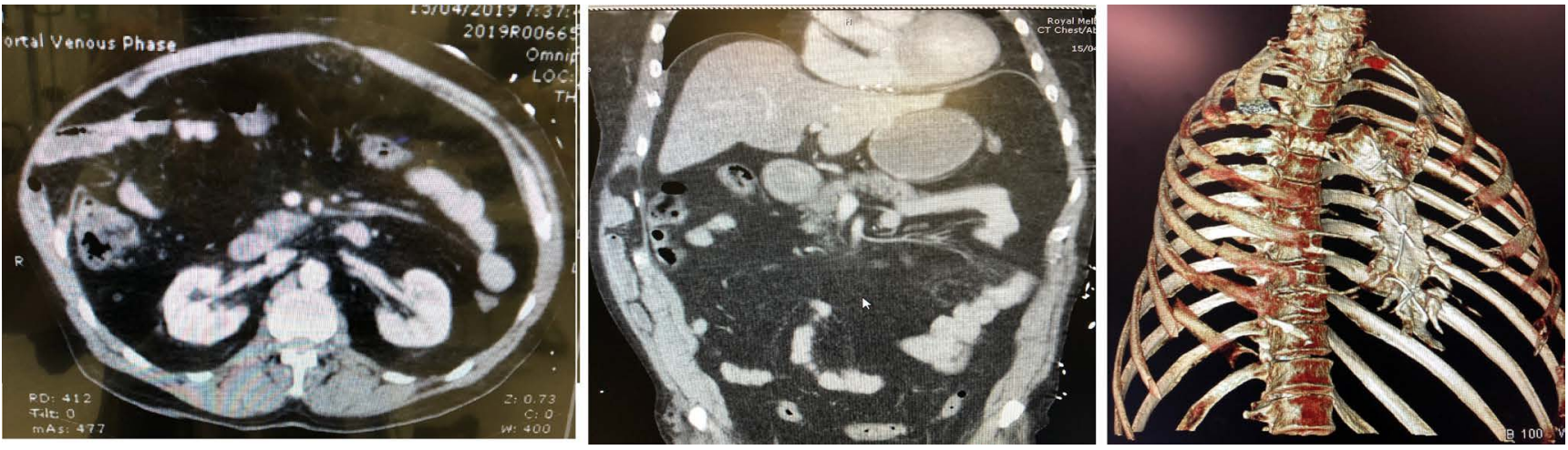

Figure 1: Transdiaphragmatic intercostal hernia and rib fractures. A) Axial CT, B) Coronal CT and C) 3D rib reconstruction.
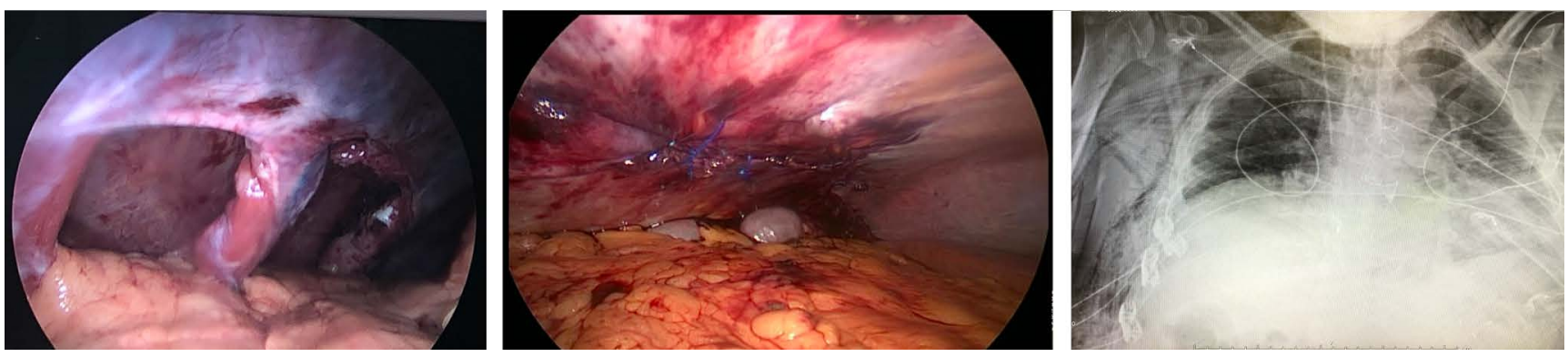

Figure 2: Intraoperative photographs of A) The hernial defect, B) Post-suturing the defect and C) A post-operative CXR showing the SSRF result.

sided rib fractures 3 to 5 using a titanium rib plating system (Figure 2). No intra-operative complications were encountered, and the patient was transferred to ICU intubated.

On day 1 post-operation, the patient was extubated and his recovery complicated by an inferior STEMI. He was commenced on dual anti-platelet agents and therapeutic heparin. His stay was further complicated by delirium and difficult glycaemic control.

\section{Discussion}

A major issue in the evidence-based management of these traumatic diaphragmatic and intercostal hernia (TDIH) injuries is the lack of clear nomenclature in this area. Dr. Gooseman's group recently proposed the Sheffield Criteria: a unifying taxonomy to clarify the assessment of injuries around the costal margin [4]. According to this system, the patient described above had a TDIH.

The Sheffield group reported four cases of TDIH, three of which occurred after coughing and one following a fall. None of those four patients had a combined thoracic and abdominal procedure, and the patient who had an abdominal approach alone had recurrence 18 months later requiring a combined mesh repair as well as costal margin plate fixation.

Macedo, et al. [5] reported three cases of transdiaphragmatic intercostal hernia occurring in association with rib fractures. All patients underwent suture and mesh repair of the hernia as well as suture re-approximation of the fractured rib ends. Long-term follow-up was not reported.

This is the first case reported in the literature of a patient undergoing both surgical stabilisation of rib fractures (SSRF) and laparoscopic mesh repair as a combined procedure in the acute setting for management of a transdiaphragmatic intercostal hernia.

\section{Conclusion}

Transdiaphragmatic hernia in association with rib fractures is rare. Acute treatment of this entity through a combined thoracic and abdominal approach allows assessment of the intra-abdominal viscera, repair of the diaphragmatic defect and reconstruction of the chest wall.

\section{References}

1. Aggarwal G, Khandelwal G, Shukla S, Maheshwari A, Mathur R, et al. (2012) Spontaneous transdiaphragmatic intercostal hernia: A rare clinical entity. Hernia 16: 113-115.

2. Lopez P, Arango J, Gallup T, Cohn S, Myers J, et al. (2010) Diaphragmatic injuries: What has changed over a 20 -year period? Am Surg 76: 512-516.

3. Unlu E, Temizoz O, Cagli B (2007) Acquired spontaneous intercostal abdominal hernia: Case report and a comprehensive review of the world literature. Australas Radiol 51: 163-167.

4. Gooseman M, Rawashdeh M, Mattam K, Rao J, Vaughan P, et al. (2019) Unifying classification for transdiaphragmatic intercostal hernia and other costal margin injuries. Eur $\mathrm{J}$ Cardiothorac Surg 56: 150-158.

5. Macedo A, Kay F, Terra R, Campos J, Aranha A, et al. (2013) Transdiaphragmatic intercostal hernia: Imaging aspects in three cases. J Bras Pneumon 39: 513-517. 